\title{
Division du travail, inégalités sociales et ordre social. Note sur les tergiversations de l'analyse durkheimienne
}

\section{Charles-Henry Cuin}

\section{OpenEdition}

\section{Journals}

Édition électronique

URL : http://journals.openedition.org/ress/378

DOI : $10.4000 /$ ress.378

ISSN : 1663-4446

Éditeur

Librairie Droz

\section{Édition imprimée}

Date de publication : 1 mars 2004

Pagination : $95-103$

ISBN : 2-600-00941-8

ISSN : 0048-8046

Référence électronique

Charles-Henry Cuin, « Division du travail, inégalités sociales et ordre social. Note sur les tergiversations de l'analyse durkheimienne », Revue européenne des sciences sociales [En ligne], XLII-129 | 2004, mis en ligne le 05 novembre 2009, consulté le 19 avril 2019. URL : http:// journals.openedition.org/ress/378; DOI : 10.4000/ress.378 
Charles-Henry CUIN

\title{
DIVISION DU TRAVAIL, INÉGALITÉS SOCIALES ET ORDRE SOCIAL. Note sur les tergiversations de l'analyse durkheimienne
}

\begin{abstract}
«Durkheim n'est visiblement pas à l'aise dans cette partie finale de son livre [le Livre III de La division du travail social], très courte par rapport aux deux précédentes et aussi bien moins élaborée [...]. De là, sans doute, un certain manque de clarté dans la construction de cette dernière partie du livre et même dans l'identification des pathologies de la société moderne.» ${ }^{1}$
\end{abstract}

Une question centrale de la sociologie durkheimienne est celle des conditions de l'ordre et de l'intégration dans un type de société caractérisé, d'une part, par un système de valeurs démocratique et, d'autre part, par la croissance de l'inégalité sociale sous l'effet du progrès inéluctable de la division du travail. Dans ses ouvrages de jeunesse, Durkheim croit trouver une réponse satisfaisante dans ce poncif classique de l'idéologie démocratique qu'est l'égalité des chances. Pourtant, au lieu de chercher à approfondir les conditions de réalisation de ce qu'il nomme lui-même l' «absolue égalité dans les conditions extérieures de la lutte», il va préférer s'orienter vers une analyse des conditions non plus socio-économiques mais socio-culturelles de l'acceptation de l'ordre social par les acteurs. En témoigne la place centrale accordée à l'éducation et, plus largement, à la socialisation dans les œuvres de maturité ${ }^{\text {. }}$

\section{L'ÉGALITÉ DES CHANCES CONTRE L'ÉGALITÉ DES CONDITIONS}

Durkheim n'est pas, à l'évidence, un sociologue de la stratification sociale. Dans La division du travail social, le thème de l'inégalité sociale occupe cependant une place privilégiée dans le traitement d'une de ses problématiques centrales - celle de la réalisation et du maintien de l'ordre social. Les « guerres de classes » et autres conflits sociaux qui agitent les sociétés industrielles n’y sont pas seulement interprétés comme une conséquence de l'anomie chronique qui y

Philippe Besnard, «Les pathologies des sociétés modernes», in Ph. Besnard, M. Borlandi et P. Vogt (Éd.), Division du travail et lien social. Durkheim un siècle après, Paris, P.U.F., 1993, pp. 197-198 passim.

Le texte qui suit est partiellement repris et adapté de Charles-Henry Cuin, «Durkheim et l'inégalité sociale: les avatars et les leçons d'une entreprise», Recherches sociologiques, 22(3), 1991, pp. 17-32. 
règne, mais aussi comme un effet du caractère «contraint» de la division du travail $^{3}$. Par là, il faut entendre que le processus de la distribution des individus dans la structure des positions sociales ne respecte pas, ou pas assez, ni les capacités propres des intéressés (leurs aptitudes et compétences) ni leurs désirs (leurs goûts et aspirations). Une telle «harmonie entre les natures individuelles et les fonctions sociales $»^{4}$ serait en effet une condition nécessaire pour que la satisfaction que chacun trouve dans l'accomplissement de son rôle social - et donc de la place qu'il occupe dans la société - l'empêche d'en désirer un autre et de mettre ainsi en cause l'ordre social établi: "Sans doute ne sommes-nous pas, dès notre naissance, prédestinés à tel emploi social; nous avons cependant des goûts et des aptitudes qui limitent notre choix. S'il n'en est pas tenu compte, s'ils sont sans cesse froissés par nos occupations quotidiennes, nous souffrons et nous cherchons un moyen de mettre un terme à nos souffrances. Or, il n'en est pas d'autre que de changer l'ordre établi et d'en refaire un nouveau.» ${ }^{5}$

Durkheim est ainsi conduit à opposer à une forme «contrainte» de la division du travail (en fait: du processus de la distribution des individus dans la structure sociale) une forme «spontanée» qui peut seule produire la solidarité sociale et prévenir les conflits sociaux. Cette spontanéité, précise-t-il, «suppose non seulement que les individus ne sont pas relégués par la force dans des fonctions déterminées, mais encore qu'aucun obstacle, de nature quelconque, ne les empêche d'occuper dans les cadres sociaux la place qui est en rapport avec leurs facultés ${ }^{6}$. Autant, dans le domaine des relations et des rapports sociaux, la société réclame une réglementation sans laquelle elle est menacée d'anomie ${ }^{7}$, autant le processus de la distribution sociale doit demeurer exempt de toute contrainte. Alors que, quelques pages plus tôt, Durkheim se faisait le chantre d'une "réglementation suffisamment développée qui détermine les rapports mutuels des fonctions ${ }^{8}$, il estime que c'est la plus totale liberté qui doit régir l'accès à ces fonctions et que rien ne doit «gêner les initiatives des individus »". Une fois la société organisée selon des règles propres à assurer l'harmonie des rapports sociaux nés de la division du travail, les destins individuels peuvent - et doivent - se déployer librement dans un espace social désormais contrôlé. La manière durkheimienne de résoudre l'antithèse classique entre individu et société est ici parfaitement balancée: au premier est due la liberté de se mouvoir dans une structure sociale convenablement organisée et d'y réaliser ses aptitudes et ses goûts, à la seconde revient l'obligation d'assurer la coordination et la complémentarité des fonctions sociales et de déterminer non pas qui doit les occuper mais comment elles doivent être remplies. La solution retenue est donc à mi-chemin entre collectivisme et individualisme.

Émile Durkheim, De la division du travail [1893], Paris, P.U.F., 1973. Il s'agit du chapitre II du Livre III, intitulé «La division du travail contrainte».

Ibid., p. 369.

Ibid., p. 368.

Ibid., p. 370.

Voir: Philippe Besnard, L'Anomie; ses usages et ses fonctions dans la discipline sociologique depuis Durkheim, Paris, P.U.F., 1987.

$8 \quad$ Ibid., p. 356.

I Ibid., p. 369. 
Elle propose le modèle d'un individu libre dans une société forte, pour le plus grand profit des deux: c'est ce que Durkheim appelle «le socialisme». «À cette condition, en effet, l'harmonie entre les natures individuelles et les fonctions sociales ne peut manquer de se produire, du moins dans la moyenne des cas. Car, si rien n'entrave ou ne favorise indûment les concurrents qui se disputent les tâches, il est inévitable que ceux-là seuls qui sont les plus aptes à chaque genre d'activité y parviennent [...]. Ainsi se réalise de soi-même l'harmonie entre la constitution de chaque individu et sa condition. $\gg^{10}$

Mais en quoi une telle procédure de distribution sociale, si visiblement favorable à la collectivité, l'est-elle également à l'individu? La réponse de Durkheim est, ici, aussi lapidaire que péremptoire: «Normalement, l'homme trouve le bonheur à accomplir sa nature; ses besoins sont en rapport avec ses moyens ». Entendons par là que, si les mérites individuels sont justement récompensés, les besoins sont satisfaits du même coup puisque les besoins correspondent aux moyens qui ont permis de réaliser les accomplissements que la société égalitaire sait reconnaître et récompenser. Bref, comme l'exprime élégamment J.-Cl. Filloux, «On a les besoins que l'on mérite !» ${ }^{11}$

La problématique durkheimienne est donc claire: comment faire en sorte que s'établisse cette "harmonie entre les natures individuelles et les fonctions sociales » jugée nécessaire à la satisfaction des individus et, par effet, à l'intégration de la société? En théorie, deux solutions distinctes se présentent. La première est celle d'un libéralisme social absolu laissant libre cours à la concurrence dans laquelle les individus s'engagent pour la conquête des différentes positions sociales. La seconde est, à l'inverse, celle d'un interventionnisme tout aussi absolu garantissant que la distribution sociale s'effectue de telle manière que les différentes positions sociales soient allouées aux individus les plus aptes à les occuper $^{12}$. Le dilemme est classique: réussissent soit les plus forts soit les plus aptes. On ne saurait mieux qualifier la solution durkheimienne de ce dilemme que de «sociale-démocrate». Cette solution est en effet celle de l'égalité des chances que Durkheim, en termes fleurant leur néo-darwinisme, définit comme une «absolue égalité dans les conditions extérieures de la lutte ${ }^{13}$. En d'autres termes, la salutaire compétition des individus les uns avec les autres dans la course pour l'accès aux positions sociales doit pouvoir se livrer sans qu'aucun des concurrents ne jouisse de quelque avantage que ce soit sur les autres. À ce compte, non seulement chacun est récompensé selon ses seuls mérites propres mais, encore et surtout, par la grâce du caractère démocratique du processus par lequel elle s'opère, le résultat de la distribution sociale se voit investi d'une forte légitimité. Non seulement l'égalité des chances permet de préserver et de rationaliser le

10 $\quad$ Ibid., p. 369.

11 Émile Durkheim, La Science sociale et l'action, Textes réunis et présentés par Jean-Claude Filloux, Paris, P.U.F., 1970, p. 24.

12 Au même moment, dans la première tradition sociologique nord-américaine, ces deux options opposées sont respectivement défendues par W.G. Sumner (What Social Classes Owe to Each Other, New York, Harper Brothers, 1883) et par L.F. Ward (La différenciation sociale et l'intégration sociale: une utopie sociologique, Paris, 1903). (Voir: Charles-Henry Cuin, Les Sociologues et la mobilité sociale, Paris, P.U.F., 1993.)

13 Émile Durkheim, De la division du travail, Op. cit., p. 371. 
caractère fonctionnel de l'inégalité des conditions, mais elle rend celle-ci légitime. Elle a donc un double mérite: d'une part elle fait coïncider aptitudes et fonctions, d'autre part elle confère à l'ordre ainsi constitué une valeur non plus seulement fonctionnelle mais également «morale».

Et c'est bien cette légitimité - plus que «le bonheur à accomplir sa nature »! qui confère à l'égalité des chances son efficacité intégratrice. De fait, lorsqu'elle est réalisée dans un contexte de chances égales, l'inégalité des conditions se voit à la fois légitimée et valorisée : légitimée car elle résulte alors de la mise en œuvre d'un idéal de justice (en l'occurrence de justice distributive), valorisée car elle récompense les mérites des uns et sanctionne négativement l'absence de mérite des autres.

La démonstration durkheimienne semble donc avoir atteint son but. Ce n'est pas l'inégalité sociale en elle-même qui est tenue pour responsable des ruptures de la solidarité sociale (et, partant, des conflits sociaux) mais le fait que les inégalités sociales ne soient pas congruentes avec les inégalités «naturelles ». L'inégalité est en effet inscrite, comme conséquence normale de la division du travail, dans la nature même des sociétés polysegmentaires ${ }^{14}$. Elle est traitée par Durkheim comme une donnée non problématique. Les classes sociales - les castes mêmes sont des modes d'organisation de la division du travail qui sont parfaitement légitimes tant qu'ils demeurent «fondé[s] dans la nature de la société », c'est-à-dire lorsque les règles de la distribution sociale ne font que structurer socialement les inégalités naturelles: «La contrainte ne commence que quand la réglementation, ne correspondant plus à la nature vraie des choses et, par suite, n'ayant plus de base dans les mœurs, ne se soutient que par la force $»^{15}$. Non, la thèse durkheimienne est bien que ce n'est pas l'inégalité des conditions qui menace la solidarité organique mais bien les conditions dans lesquelles cette inégalité d'une part se constitue et, d'autre part, se maintient. Et l'égalité des chances est l'instrument par lequel peut se réaliser cette adéquation entre ce que les hommes sont «naturellement» et ce qu'ils deviennent «socialement».

On s'attendrait donc à ce que Durkheim développe et approfondisse une analyse des conditions de réalisation de cette providentielle égalité des chances. Celui-ci n'identifie pourtant comme seul et unique obstacle à cette réalisation que l'institution de l'héritage - plus précisément de l'héritage patrimonial: «[...] alors même qu'il ne reste, pour ainsi dire, plus de trace de tous ces vestiges du passé, la transmission héréditaire de la richesse suffit à rendre très inégales les conditions extérieures dans laquelle la lutte s'engage $»^{16}$.

Pourtant, plus on avance dans la lecture de La Division du travail social - ou même du Socialisme - et plus il devient clair que, sous l'expression d' "égalité dans les conditions extérieures de la lutte», il y a davantage que la seule notion d'égalité des chances dans l'accès aux positions sociales. Il faut en effet ajouter à la dimension distributive de ce principe de justice une dimension rétributive selon laquelle les différentes fonctions sociales ne doivent pas seulement être librement

14 «[... les progès de la division du travail impliquent [...] une inégalité toujours croissante [...]», Ibid., p. 371.

$15 \quad$ Ibid., p. 370.

16 Ibid., pp. 371-372. 
accessibles par chacun indépendamment de son origine sociale mais doivent en outre recevoir des gratifications matérielles et symboliques proportionnelles aux services rendus. Durkheim précise en effet que «cette égalité dans les conditions extérieures de la lutte [...] consiste non dans un état d'anarchie qui permettrait aux hommes de satisfaire librement toutes leurs tendances bonnes ou mauvaises, mais dans une organisation sociale où chaque valeur sociale, n'étant exagérée ni dans un sens ni dans l'autre par rien qui lui fût étranger, serait estimée à son juste prix $\gg^{17}$.

Durkheim est infiniment plus disert sur les conditions de la réalisation de cette dernière exigence que sur celles de la précédente. Pour assurer l'égalité contractuelle des rapports sociaux, il faut en effet que les valeurs échangées par les individus (des biens et des services les uns contre les autres) soient équivalentes, c'est-à-dire que le prix de l'objet échangé soit «en rapport avec la peine qu'il coûte et les services qu'il rend». Si cette valeur n'est pas «mathématiquement» calculable, la «conscience publique» (ou encore l' «opinion») possède un sentiment assez précis de cette valeur et est donc en mesure de juger sainement du degré d'équité de l'échange. Mais ce n'est heureusement pas tout: la thèse durkheimienne est que, si le consentement des contractants est libre de toute pression extérieure (s'ils sont «placés dans des conditions extérieures égales ${ }^{18}$ ), alors l'échange est équitable et les individus ne reçoivent qu'en fonction du coût réel de l'objet échangé. Si, au contraire, «une classe de la société est obligée, pour vivre, de faire accepter à tous prix ses services, tandis que l'autre peut s'en passer grâce aux ressources dont elle dispose et qui pourtant ne sont pas nécessairement dues à quelque supériorité sociale, la seconde fait injustement la loi à la première. Autrement dit, il ne peut pas y avoir des riches et des pauvres de naissance sans qu'il n'y ait des contrats injustes $»^{19}$.

Ainsi, l'essentiel de l'analyse de l'analyse durkheimienne des causes de conflits sociaux tenant aux modalités selon lesquelles les mérites individuels sont à la fois reconnus (par la manière dont le processus de la distribution sociale s'effectue) et récompensés (par le résultat de ce processus en termes de stratification sociale) tient dans la dénonciation de la seule institution de l'héritage patrimonial, qui interdit l'égalité des chances et rend les rapports sociaux inéquitables. Le mérite de cette analyse n'est cependant pas mince, dans la mesure où elle parvient à dépasser l'aporie consubstantielle à toute idéologie de l'égalité des chances: l'égalité des chances débouchant sur une inégalité des conditions (qui, dans le même temps, en légitime le principe), il suffit de faire en sorte que les conditions d'arrivée d'une génération ne constituent les conditions de départ de la suivante. C'est ce que permettrait, en brisant le cercle vicieux de la reproduction socioéconomique, la suppression de l'héritage patrimonial!

\footnotetext{
Émile Durkheim, De la division du travail, op. cit., p. 371.

$18 \quad$ Ibid., p. 377.

$19 \quad$ Ibid., p. 378.
} 


\section{LES APORIES \\ DE LA SOLUTION SOCIO-ÉCONOMIQUES ET LE CHOIX DE LA SOLUTION SOCIO-CULTURELLE}

Il est cependant douteux qu'il suffise de supprimer les inégalités économiques de «naissance» pour supprimer du même coup les «contrats injustes». D'une part, il existe, à côté de la seule hérédité économique, bien d'autres hérédités - en particulier sociales et culturelles - susceptibles d'altérer la «spontanéité » de la distribution sociale. D'autre part, les rapports sociaux ne sont pas affectés seulement par les situations économique de départ dans lesquelles se concluent les contrats mais aussi, et plus significativement encore, par les l'ensemble des inégalités plus ou moins acquises dont les individus sont affectés tout au long de leur carrière. Ce qui rend les contrats «injustes », c'est l'inégalité même des contractants, et pas seulement celle qui résulte de la transmission héréditaire des biens. En outre, dans un cours sur la famille conjugale professé en 1892, Durkheim montrait déjà l'extrême difficulté qu'il y aurait à supprimer une institution jouant un rôle si efficace de stimulant pour le travail et pour la réussite individuelle ${ }^{20}$ !

Mais, la suppression de l'héritage assurerait-elle l'égalité des chances, il resterait que l'instauration de cette dernière est loin de constituer une solution efficace à la problématique durkheimienne du maintien de l'ordre social par la loyauté des acteurs. De fait, la distribution «naturelle» des talents et des aspirations parmi les individus a bien peu de chances (et, pour dire vrai, aucune) de correspondre à celle des positions sociales définies par la division du travail. La raison en est fort simple: la première de ces distributions est aléatoire tandis que la seconde est «donnée »! Dans ces conditions, comme R. Boudon l'a magistralement et définitivement montré dans ses travaux sur la mobilité sociale ${ }^{21}$, l'égalisation des chances peut fort bien n'avoir pas les effets méritocratiques attendus et, donc, les effets d'intégration par justice distributive interposée prévus par Durkheim.

Ainsi le bilan de la mise en œuvre durkheimienne de la problématique en termes d' «égalité des chances » apparaît bien précaire - tant au plan des conditions de son application pratique qu'à celui des effets qui en sont attendus. Aussi peut-on, avec un lecteur aussi attentif qu'A. Pizzorno, s'étonner «de voir comment une pensée sociologique si pénétrante, après avoir recouru à ce concept d'égalité à un point fondamental et critique du système, oublie de se demander quelle en est la signification sociologique $»^{22}$. Mais s'agissait-il vraiment d'un oubli?

Quelques années plus tard, dans les dernières pages du Socialisme, Durkheim allait en effet apporter une réponse bien différente de la précédente à la question agitée dans le chapitre sur «La division du travail contrainte »: «Ce qu'il faut pour

Émile Durkheim, «La famille conjugale» [1892], in Textes 3. Fonctions sociales et institutions, Paris, Minuit, 1975, p. 47.

21 Raymond Boudon, L'Inégalité des chances. La mobilité sociale dans les sociétés industrielles, Paris, A. Colin, 1973.

22 Alessandro Pizzorno, «Lecture actuelle de Durkheim», Archives européennes de sociologie, 1963, IV, pp. 1-36. 
que l'ordre social règne, c'est que la généralité des hommes se contentent de leur sort; mais ce qu'il faut pour qu'ils s'en contentent, ce n'est pas qu'ils aient plus ou moins, c'est qu'ils soient convaincus qu'ils n'ont pas le droit d'avoir plus. [...] S'il ne sent pas au-dessus de lui une force qu'il respecte et qui l'arrête, qui lui dise avec autorité que la récompense qui lui est due est atteinte, il est inévitable [que l'individu] réclame comme lui étant dû tout ce qu'exigent ses besoins et, comme dans l'hypothèse ces besoins sont sans frein, leurs exigences sont nécessairement sans bornes $»^{23}$. Le changement de ton est radical. Ici, les «besoins » de l'individus ne sont plus naturellement «en rapport avec ses moyens»; ils sont au contraire, comme décrits dans Le Suicide, infiniment extensibles et, de ce fait, insatiables aussi longtemps que l'intériorisation de normes sociales adaptées ne parvient à les réguler et, donc, à créer la possibilité de leur satisfaction ${ }^{24}$. Comme on peut s'en convaincre par la lecture des textes ultérieurs sur l'éducation, Durkheim vient de marquer qu'il abandonne la solution socio-économique de la question de l'ordre social au bénéfice d'une solution socio-culturelle.

De fait, contrairement à toute attente, la conception durkheimienne de l'éducation ne fait pas de l'institution scolaire un instrument d'égalisation des chances permettant la réalisation d'une distribution sociale méritocratique. Le rôle de l'école est d'abord de répondre à une demande structurelle déterminée en amont du processus éducatif par l'état de la division du travail et, dans ce but, d'y conformer les individus qui lui sont confiés. Elle cherche moins à sanctionner les mérites individuels qu'à produire des individus adaptés aux besoins collectifs, c'est-à-dire à la demande sociale. À cet égard, les propos de Durkheim sont sans ambiguïté: «Bien loin que l'éducation ait pour objet unique ou principal l'individu et ses intérêts, elle est avant tout le moyen par lequel la société renouvelle perpétuellement les conditions de sa propre existence $»^{25}$.

On est donc bien loin de la thèse selon laquelle la seule évaluation des mérites individuels dans une situation d'égalité des chances permettrait de réaliser «l'harmonie entre les natures individuelles et les fonctions sociales». Ici, plus un mot sur l'égalité des chances: l'École, qui a pour fonction première (comme P.A. Sorokin le soulignera plus $\operatorname{tard}^{26}$ ) de distribuer dans les différentes positions sociales des individus aux caractéristiques appropriées, a donc essentiellement pour charge de leur donner les compétences nécessaires à leur efficacité dans leurs fonctions respectives - bref, à «susciter et [...] développer chez l'enfant un certain nombre d'états physiques, intellectuels et moraux que réclament de lui la société politique dans son ensemble et le milieu spécial auquel il est particulièrement destiné» ${ }^{27}$. Évidemment, rien n'est dit des raisons pour lesquelles un indi-

23 Émile Durkheim, Le Socialisme, sa définition, ses débuts, la doctrine saint-simonienne [1928], Paris, P.U.F., 1971, p. 227.

${ }_{24}$ Émile Durkheim, Le Suicide. Étude de sociologie [1897], Paris, P.U.F., 1973. (Sur cet aspect de l'analyse durkheimienne, voir: Ch.-H. Cuin, «Durkheim et la mobilité sociale», Revue française de sociologie, 1987, XXVIII, 1, pp. 43-65.)

25 Émile Durkheim, Éducation et sociologie [1922], Paris, P.U.F., 1966, p. 91.

26 Pitirim A. Sorokin, Social and Cultural Mobility [1927], Glencoe, Illinois, The Free Press, 1959. Voir également: Charles-Henry Cuin, «Sorokin et le 'Social Mobility' de 1927: naissance et mise en œuvre d'une problématique sociologique», L'année sociologique, 38, 1988, p. 275-308.

27 Émile Durkheim, Éducation et sociologie, op. cit., p. 41 (c'est nous qui soulignons). 
vidu donné serait «destiné » à embrasser telle carrière plutôt que telle autre ! Il ne peut plus, en effet, s'agir de goûts ou autres aptitudes innés: depuis Le Suicide, le lecteur de Durkheim sait qu'il n'y a d'autre «nature humaine» que celle que la société, par la socialisation, crée de toutes pièces en nous.

Dans cette perspective, il devient alors évident que l'égalité des chances n'a plus grand rôle à jouer. L'essentiel étant de placer les individus convenables là où la société réclame qu'ils soient placés, c'est cette «demande» qui doit être satisfaite en priorité - et quelles que soient a priori les exigences de l'offre individuelle.

Mais que devient alors l'impérieuse nécessité de faire en sorte que «la généralité des hommes se contentent de leur sort »? C'est là que l'institution éducative révèle le caractère providentiel de son action - en forme de véritable «sociodicée $\gg^{28}$. De fait, si le système éducatif joue un rôle fonctionnel d'induction («susciter») et de développement des qualités individuelles diverses et variées que requièrent les fonctions créées par la division du travail, il joue aussi, et dans le même temps, un rôle moral de socialisation, d'adaptation et d'intégration de l'individu. S'il parvient à réaliser l'«harmonie» attendue entre ce que les individus sont moralement et ce qu'ils font socialement - entre aspirations et destins individuels -, ne devient-il pas alors assez indifférent que cette distribution sociale résulte d'une situation d'égalité des chances? Le discours durkheimien est ici sans détour: "L'homme que l'éducation doit réaliser en nous, ce n'est pas l'homme tel que la nature l'a fait, mais tel que la société veut qu'il soit; et elle le veut tel que le réclame son économie intérieure ${ }^{29}$.

En dernière analyse, Durkheim semble donc avoir estimé que le «scandale moral» que constitue l'inégalité des chances serait plus efficacement évité en préparant les individus à ce qu'ils seront (et ce qu'ils seront reproduira sans doute ce qu'ils sont, c'est-à-dire leur origine sociale...) qu'en leur permettant de devenir ce qu'ils ont la capacité, la volonté ou le goût d'être. Cela expliquerait en effet, d'une part, la précarité de la réflexion de notre auteur sur les conditions de l'égalisation des chances sociales et, d'autre part, l'accent mis par celui-ci sur le rôle essentiellement socialisateur et intégrateur de l'institution scolaire, au détriment de son rôle de promotion sociale des individus.

La raison d'une telle évolution tient sans doute au fait que, dans cette œuvre de jeunesse qu'est La Division du travail social, le paradigme durkheimien n'est pas encore entièrement élaboré. Et ce sont ces incomplétudes qui, sans doute, ont conduit Durkheim à se fourvoyer dans des pistes de recherches dont il a dû constater trop tard qu'elles conduisaient à des impasses. Si la thèse selon laquelle les conflits sociaux ont leur source essentielle dans l'illégitimité de l'ordre social (i.e., ce n'est pas la nature objective des rapports sociaux qui risque rompre le consensus mais le caractère défavorable de la perception qu'en ont les acteurs) reste inchangée, Durkheim est rapidement passé d'une conception selon laquelle

\footnotetext{
28 Ce thème, on le sait, occupe une place centrale dans les théories françaises de la «reproduction sociale» (Voir: Pierre Bourdieu et Jean-Claude Passeron, La Reproduction; éléments pour une théorie du système d'enseignement, Paris, Ed. de Minuit, 1970 et Christian Baudelot et Roger Establet, L'École capitaliste en France, Paris, Maspero, 1971.)

29 Émile Durkheim, Éducation et sociologie, op. cit., p. 90.
} 
cette légitimité pouvait être obtenue par l'organisation démocratique du système social à une conception plus radicale pour laquelle le même résultat serait plus efficacement atteint par l'inculcation systématique et institutionnalisée de valeurs et de normes - bref, d'une solution socio-économique à une solution socio-culturelle.

Université Victor Segalen - Bordeaux 2 c.cuin@wanadoo.fr 